\title{
Plasmodial incompatibility in the myxomycetes: a review
}

\author{
Clark $\mathbf{J}^{1^{*}}$ and Haskins $\mathbf{E F}^{2}$ \\ ${ }^{1}$ Department of Biology, University of Kentucky, Lexington, Kentucky 40506 - jimc1939@ frontier.com \\ ${ }^{2}$ Department of Biology, University of Washington, Seattle, Washington 98195 - edward.haskins@ comcast.net
}

Clark J, Haskins EF 2012 - Plasmodial incompatibility in the myxomycetes: a review. Mycosphere 3(2), 131-141, Doi 10.5943/mycosphere/3/2/3

Two myxomycete phaneroplasmodia of the same species undergo somatic fusion only if they are phenotypically identical for a complex genetic incompatibility system. This system consists of a three tiered polygenic complex with dominant and recessive alleles. Thus, plasmodia must be phenotypically identical for approximately 16 loci in order to fuse (CC and $\mathrm{Cc}$ are phenotypically identical, but different from cc). The first level of the system (having a minimum of seven Fus loci) controls membrane fusion, and it apparently prevents fusion unless the two plasmodia have identical membrane or slime sheath components. The second level (having a minimum of six $\mathrm{Cz}$ loci) produces a rapid lysis of a small mixed region, of the two plasmodia, if membrane fusion has occurred. This lysis is directional in that it targets the recessive phenotype, and it is apparently triggered by some pre-formed substances when they come into contact with a different plasmodium. The third level (having a minimum of three Let loci) comes into play if membrane fusion occurs and there is no rapid lysis of the mixed plasmodium. It produces a slow lethal reaction, which targets and degrades the nuclei of the recessive phenotype. This reaction occurs over a period of five to twenty hours and requires the synthesis of new RNA and proteins. Since, this complex system produces a minimum of 65,536 different incompatibility phenotypes, it is highly unlikely that any two phaneroplasmodia will undergo a successful fusion unless they are very closely related. Species with aphaneroplasmoida apparently have a similar system, but species with small protoplasmodia do not appear to undergo any type of plasmodial fusion.

Key words - incompatibility - myxomycetes - self-recognition - somatic-fusion

\section{Article Information}

Received 21 February 2012

Accepted 28 February 2012

Published online 25 March 2012

Corresponding author: Jim Clark - jimc1939@frontier.com

\section{Introduction}

The ability to distinquish self from nonself is necessary for any organism that wishes to maintain its individuality. This is especially difficult in the myxomycetes with a phaneroplasmodial vegetative stage, since it is a large coenocytic cell covered only by a slime sheath (Haskins \& Hinchee 1974). This plasmodium is mobile and forms a continuously modified reticulate structure by the fusion of different regions. Thus, there is little, or no, physical barrier to fusion either within a single phaneroplasmodium or between two different phaneroplasmodia.

Plasmodial fusion, and non-fusion, in the myxomycetes was observed in the early studies of this group (Cienkowski 1863), and it was noted by de Bary (1887) that neither he nor his students had ever observed a fusion between the plasmodia of two different species of myxomycetes. On the 
other hand, Massee (1892) believed that the plasmodia of different species could sometimes fuse. Some years later, Torrend (1907) suggested that plasmodia which fused when brought into contact with each other could be assumed to belong to the same species, whereas if they failed to fuse they belonged to separate species. However, Brandza (1927) found that some plasmodia from the same species failed to fuse with each other, and that they should therefore be considered to be different strains of the same species. Skupienski (1934) working with Didymium iridis (as D. xanthopus) felt that he had demonstrated the existence of physiological races, and in 1939 he drew the same conclusion for Didymium squamulosum (Skupienski 1939). Later workers, Gray (1945) working with Physarum polycephalum, and Alexopoulos \& Zabka (1962), and Mukherjee (1965) working with $D$. iridis, also used the term physiological race to distinquish plasmodia of the same species that did not fuse with each other. These conclusions were in keeping with the techniques then available and with the kind of genetically undefined material which they used.

\section{Genetic analysis of incompatibility systems}

Three species, with large phaneroplasmodia, have been the subjects of a genetic analysis of their plasmodial incompatibility system: Didymium iridis, Physarum policephalum, and Physarum cinereum.

\section{Didymium iridis}

The starting point for the genetic study of plasmodial fusion is Collins (1966) who in dealing with a Honduran isolate of Didymium iridis, produced plasmodia by crossing a number of different myxamoebal clones and tested them against each other for fusion. When he found that these crosses produced a number of different fusion classes, he concluded that plasmodial fusion was controlled by a specific genetic system, as opposed to physiological race differences that had been postulated by earlier workers. This original work was followed by the research of his two students (Clark and Ling) who began their work in his laboratory. Clark (Collins
\& Clark 1966; 1968; Clark \& Collins 1972, 1973) using the Honduran isolate uncovered a polygenic system of five loci (C/c, D/d, E/e, F/f, G/g) with dominant and recessive alleles that controlled plasmodial fusion. For any two plasmodia to fuse, they had to be phenotypically identical at all five loci (Cc and CC are phenotypically identical, but different from $\mathrm{cc}$ ). Of course genotypically identical plasmodia will always fuse, since they are also phenotypically identical. Clark \& Collins found that a $G$ locus difference apparently prevented any fusion, but that differences at the other four loci allowed a limited fusion that was rapidly terminated by a lethal reaction in the mixed cytoplasm region (produced a clear zone of coagulated material). They also concluded that the $\mathrm{G}$ locus was linked to the mating type locus, that the $\mathrm{C}$ and $\mathrm{E}$ loci were linked, and that the D and $F$ loci were also linked to each other. Ling (Ling \& Collins 1970a, 1970b, Ling 1972) using a Panamanian isolate also uncovered a polygenic system of six loci (Q/q, R/r, S/s, T/t, U/u, V/v) with dominant and recessive alleles that phenotypically controlled plasmodial fusion. In this isolate none of the six loci allowed a limited fusion to occur, and the $S$ locus was linked to the mating locus, $\mathrm{U}$ and $\mathrm{V}$ were linked, and $\mathrm{Q}$ was linked to a plasmodial colour gene. The plasmodial fusion genes of these two isolates (Hon and Pan) were then correlated in several papers (Collins \& Ling 1972, Ling \& Ling 1974, Ling \& Clark 1981) where it was found that there were at least 13 loci controlling plasmodial fusion in the two isolates of this species. These loci were correlated and re-designated to reflect their function: fusion loci (Fus) that prevent fusion and clear zone loci $(\mathrm{Cz})$ that allow limited fusions that result in a small clear zone of coagulated material. The new designations and their old designations are as follows: Fus1 (Q), Fus2 (R), Fus3 (S or G), Fus4 (T), Fus5 (U), Fus6 (V), Fus7 (temporarily called Y by Ling), Cz1 (D and temporarily called W), Cz2 (temporarily called X), Cz3 (temporarily called $\mathrm{Z}$ ), $\mathrm{Cz} 4$ (F and temporarily called L), Cz5 (C), and Cz6 (E), with linkages between Fus1 and the plasmodial colour marker, Fus3 and the mating type locus, Fus5 and Fus6, Cz1 and Cz4, and Cz5 and Cz6. Clark \& Collins (1978) also 
looked at the genetics of plasmodial fusion in a Kentucky isolate of $D$. iridis, which, at that time, they considered to be Didymium nigripes, but is now identified as a sibling biological species of D. idiris (does not mate with the Honduran and Panamanian isolates). They found a polygenic system with two loci $(\mathrm{C} 1 / \mathrm{c} 1, \mathrm{C} 2 / \mathrm{c} 2)$ with dominant and recessive alleles that phenotypically control plasmodial fusion, in which both loci allowed small fusions areas that produced clear zones. The ability to dissect a polygenic system with 13 loci in a diploid organism is probably unique to the myxomycetes (and some yeast), and is only possible due to the nature of its gametes. The gametes are directly converted from vegetative myxamoebal cells, and thus clonal populations of myxamoebae derived from isolated single spores are in effect clonal gametes which can be used in multiple crosses to build up genetic information on that clone. This can be a slow and laborious process, but it does allow for the investigation of many genes at the same time (see Clark \& Haskins 2011).

\section{Physarum polycephalum}

The Carlile \& Dee (1967) report, using the Wisconsin isolate, was the first genetic study of incompatibility in $P$. polycephalum. They found that fusion could only occur when plasmodia were identical for two co-dominant alleles (f1 and f2) at a single locus: f1f1 plasmodia would not fuse with f1f 2 or $\mathrm{f} 2 \mathrm{f} 2$ plasmodia, f1f 2 plasmodia would not fuse with f1f1 or $\mathrm{f} 2 \mathrm{f} 2$ plasmodia, and $\mathrm{f} 2 \mathrm{f} 2$ plasmodia would not fuse with f1f1 or f1f2 plasmodia. This work was continued by Poulter \& Dee (1968), who included the Indiana isolate in their studies. They identified two additional alleles ( $\mathrm{f} 3$ and $\mathrm{f4}$ ) of the fusion locus which were also co-dominant in their actions: this gave ten possible genotypes in which each genotype should not fuse with any of the other nine genotypes, however, for some unexplained reason the f3f3 and $\mathrm{f} 4 \mathrm{f} 4$ genotypes did undergo fusion. Wheals (1970) reported that Poulter had also found a second locus that controlled plasmodial fusion in the Indiana isolate, this locus had two alleles (n1 and n2) which displayed a dominant (n2) and recessive (n1) allelic relationship, so that $n 2 n 2$ and $\mathrm{n} 2 \mathrm{n} 1$ plasmodia fused, but neither would fuse with n1n1 plasmodia. Wheals also found that the Colonia (German) isolate that he was working with had $\mathrm{f} 1$ and $\mathrm{n} 2$ compatibility alleles. Carlile \& Dee (1967) had observed that some plasmodia which were homozygous for the f locus, and thus could fuse, underwent a lethal reaction (coagulated region) which killed a large part of the fused plasmodium. Carlile (1976), using the progeny of one of these plasmodia studied the genetics that controlled this lethal reaction; and found that it was controlled by a polygenic system of three loci displaying dominant and recessive alleles (letA/a, letB/b, letC/c). Therefore, any two plasmodia that differed phenotypically for one or more of these loci would produce a lethal reaction. He also reported that the letA and letC loci were linked.

Meanwhile Collins \& Haskins (1970, 1972) using the Iowa (= PpII) isolate of $P$. polycephalum found that plasmodial fusion in this isolate was controlled by a polygenic system of four loci with dominant and recessive alleles $(\mathrm{C} / \mathrm{c}$, $\mathrm{D} / \mathrm{d}, \mathrm{E} / \mathrm{e}, \mathrm{F} / \mathrm{f}$ ). In this system any two plasmodia which differed phenotypically for any one or more loci would not undergo fusion. Collins (1972) continued this study using the same Indiana (= Turtox) isolate that Poulter and Dee had used. However, his results differed from theirs, in that he again found a polygenic system of four loci (C/c, D/d, E/e, F/f) displaying dominant and recessive alleles. He did not try to correlate these loci to those identified in the Iowa strain, so that they could be the same loci, partially the same loci (the most likely situation), or completely different loci. It is difficult to see how the results of Collins (1972) and the Poulter $\&$ Dee (1968) can be reconciled, since they were both working with the same Indiana isolate. However, since Collins' result agrees with all of the other genetic reports on the control of plasmodial fusion (polygenic system with phenotypic fusion), it is likely that there was a mistake made in the analysis that found codominant alleles by Dee's group.

\section{Physarum cinereum}

A genetic analysis (Clark 1977) of plasmodial fusion in a Venezuelan isolate of 
Physarum cinereum uncovered a polygenic system of two loci $(\mathrm{C} 1 / \mathrm{c} 1, \mathrm{C} 2 / \mathrm{c} 2)$ displaying dominant and recessive alleles. Therefore its system is essentially the same as the $D$. iridis and $P$. polycehalum systems, where two plasmodia will fuse only if they are phenotypically identical at these loci.

\section{Fusion reactions}

When two different plasmodia come into contact with each other, a number of different events can occur: they can undergo complete fusion to form a single entity; they can fuse to form a single plasmodium that loses one set of nuclei after several hours or days; they can undergo a fusion that is quickly terminated by the lyses of the mixed area; or they can remain as separate plasmodia which either ignore each other or undergo a competitive elimination.

\section{Complete fusion}

When two genetically identical plasmodia, or those that are phenotypically identical at all of the relevant loci, meet they quickly fuse and thereafter function as a single entity. The plasmodia push up against each other and thin out the slime sheath so that the plasma membranes can touch, form bridges, and develop vein-like channels which allows the two plasmodia to mix. A number of studies have shown that this mixing is complete (Kerr 1963, Miller et al. 1964, McCormick 1974) and is not affected by nutrition (Kerr \& Waxlax 1968). However, fusion may be delayed for some time (Lane \& Carlile 1979) if one of the plasmodia is undergoing mitotic division (all of the nuclei in the plasmodium undergo periodic synchronized divisions).

\section{Slow lethal reactions}

Slow lethal reactions were probably first observed by Seifriz (1944) who though that they were caused by an exotoxin. They occur in heterotkaryotic plasmodia several hours or more after plasmodial fusion has occurred and are manifest by either a lytic reaction which kills a large portion of the heterokaryon several hours after fusion with only one of the original nuclear types surviving, or by the elimination of one set of nuclei from the heterokaryon approximately 24 hours after fusion without outward evidence of lysis. This reaction has been intensely studied in the Wisconsin isolate of Physarum polycephalum using two plasmodia which produce a large lethal reaction. Carlile (1972) determine that two inbred plasmodia designated 15 (= killer) and 29 (= sensitive) underwent a lethal reaction 4 to 5 hours after fusion with the lytic zone delimited from the active plasmodium by a thick membrane, and that the sensitive plasmodia and its nuclei was generally completely eliminated. He also found that under starvation conditions the visible lethal reaction did not occur, but that nuclear elimination still occurred within 24 hours. Genetic analysis (Carlile 1976) of this reaction indicated that it was controlled by a polygenic system of three loci with dominant and recessive alleles that caused the reaction if two plasmodia differed phenotypically at one or more of these loci. Ultrastructure studies (Border \& Carlile 1974, Lane \& Carlile 1979) using autoradiography to mark the sensitive strain's nuclei found that these nuclei were selectively damaged (chromatin condensation and nucleolar segregation), enclosed in vacuoles, and eliminated. There was also a general increase in endoplasmic reticulum vesicles, and nuclear enlargement and fusions. A physiological investigation of the lethal reaction by Schrauwen $(1979,1981,1985 a, 1985 b)$ found that the reaction was not caused by extra-cellular or preformed intra-cellular compounds, but required the synthesis of RNA and protein after the fusion occurred. Since the nuclei of both the killer and sensitive strains must be blocked to prevent the reaction, it is likely that the lytic compound is produced by both nuclear types. The target of the reaction is apparently the membranes (changes in phospolipid synthesis occur) and the DNA of the sensitive strain (DNA fragmentation occurs). Heterokaryon breakdown has also been studied In $P$. polycephalum by Dee and Anderson (1984) using plasmodia genetically identical except for several markers (a plasmodial colour gene, an amino acid requirement gene, and haploid and diploid nuclei). They found that when two haploid plasmodia were fused the heterokaryon 
was stable, but when a diploid and haploid plasmodium were fused, the diploid nuclei were lost; apparently the diploid nuclei were diluted out during plasmodial growth. Slow lethal reactions, in the form of an elimination of one nuclear type from a heterokaryon, is also present in Didymium iridis. Kerr (1965) using a mutant plaque marker, and Clark and Collins (Collins \& Clark 1968, Clark \& Collins 1972)using both a plasmodial colour marker and a mating type difference marker, found that the nuclei of one of the original plasmodia types did not survive even when the two plasmodia were highly inbred. In the case of the mating type heterokaryon, it is possible that the mating type allele acts either directly as a incompatibility gene or is closely linked to one. Clark \& Hakim (1980) found that no nuclear killing occurred after transfilter contact between two heterokaryon incompatible plasmodia (plasmodial on opposite sides of a one micron pore size filter undergo cytoplasmic fusion but the nuclei cannot pass through the pores). Apparently, the production of the lytic compound requires a close contact between the different nuclei.

\section{Fast lethal reaction}

The fast lethal reactions have been studied in Didymium iridis, where Ling and Clark (1981) found a total of six $\mathrm{Cz}$ loci in the Honduran and Panamanian isolates. These loci act polygenically and each has a dominant and recessive allele. Two plasmodia that are phenotypically identical for the Fus loci (fusion can occur) but phenotypically different at one or more $\mathrm{Cz}$ loci will fuse, but the mixed area will undergo a lytic reaction within seconds to form a clear zone which is walled off from the rest of the plasmodia by a thick membranous structure (Upadhyaya \& Ling 1976). This reaction is directional, in that the nuclei from the recessive plasmodium are destroyed (Ling \& Upadhyaya 1974), and the clear zone usually occurs in the recessive plasmodial region (Clark \& Collins 1972). The reaction is also additive, when the two plasmodia are different at two or more $\mathrm{Cz}$ loci, the reaction is faster and thus smaller than that produced by either of the two loci acting alone (Clark \&
Collins 1972). Electron microscopic investigations of the clear zone (Upadhyaya \& Ling 1972, 1976) found that the zone was delimited by a thick membranous structure, that it contained many lipid droplets, and clumped organelles, but that the nuclei enclosing vesicles, of the slow reaction, were not present. Similar fast lethal reactions were detected in Physarum cinereum (Clark 1977) and in a sibling biological species (Kentucky isolate which does not mate with the Honduran and Panamanian isolates) of $D$. iridis (Clark \& Collins 1978).

\section{Non fusion reactions}

Most work on non-fusion has also been done using Didymium iridis (Collins \& Clark 1968, Clark \& Collins 1972, Ling \& Ling 1974, Ling \& Clark 1981). A total of seven polygenic loci with dominant and recessive alleles, phenotypically determine if two different plasmodia can fuse. Presumably these plasmodia differ for some cell surface factor, but the possibility that these loci are very strong fast reactions (terminate any fusion before it becomes visible) can't be dismissed. Ross \& Shipley (1973) conducted some preliminary experiments which seemed to indicate that a factor was produced during the myxamoebae to plasmodia conversion, which changed the membrane from being able to fuse with other myxamoebae to a state which did not recognize myxamoebae as fusion partners, and in fact engulfed then as food. Also, Jeffery \& Rusch (1974) were able to fuse incompatible $P$. polycephalum plasmodia (presumably differing for fusion loci) by disrupting the two plasmodia into small nucleated pieces and mixing them together. Some of the reorganized plasmodium then displayed the combined fusion phenotype of the two original plasmodia (could not fuse with either of the original two plasmodia). Similarly, Clark (1984) using a three clone mating procedure (when log growth phase myxamoebae are mixed together they undergo mass fusions) with clones selected so that two diploid nuclear types could be produced having either the $\mathrm{cDEFG}$ or CdEFG incompatibility phenotype, found that the resulting plasmodium had a CDEFG phenotype. 
Some of these plasmodia retained the all dominant phenotype until they died, while others reverted to the CdEFG phenotype some time after reaching a large size. Apparently plasmodia express the phenotype of all of the genes present at the time of plasmodial formation and are tolerant of any incompatibility genes which remain in the active plasmodium. Non fusion reactions have also been reported in $P$. polycephalum (Carlile \& Dee 1967, Poulter \& Dee 1968), and Badhamia utricularis (Carlile 1974).

\section{Plasmodial competition}

When plasmodia are cultured from the wild, on agar or in moist chambers, a number of different plasmodia may be present, however, after a period of time only a single plasmodium usually is present (especially if the plasmodia are incompatible plasmodia of the same species). Therefore, competition between plasmodia in these restricted circumstances must take place. This is illustrated by mass-spore cultures of heterothallic species, where the germinated myxamoebae should cross in many different combinations in terms of the plasmodial incompatibility loci, to give all of the possible phenotypic fusion classes. However, in every mass-spore culture there is only a single fusion class plasmodium, which has the all dominant fusion phenotype (Collins 1966, Collins \& Clark 1968). Thus, plasmodial competition, at least between plasmodia of the same species, appears to be mediated by the incompatibility loci. This hypothesis was tested using the Honduran isolate of $D$. iridis by Clark (1980a, 1980b). In one series of tests, plasmodia which differed phenotypically for the C, D, E, or F incompatibility loci were produced and the cytotoxic rating difference between each possible paired phenotype was calculated (the locus $\mathrm{G}$ is a Fus locus and was not included in the calculation). Equal sized inocula of two different plasmodia were then paired in a Petri dish and maintained until there was only one remaining plasmodium. The survivor in most cases could be predicted beforehand by the cytotoxicity rating differences of the two plasmodia. In a second series of test, four myxamoebal clones (two of each mating type) were crossed (all four clones placed in the same Petri dish). These clones were selected so that four different possible incompatibility phenotype plasmodia could be produced in each Petri dish. The crosses were then allowed to form plasmodia and maintained until only one plasmodium remained. Again the survivor could generally be predicted by the incompatibility rating difference between the four possible plasmodia. Thus, under confined conditions, the incompatibility phenotype of the plasmodia controls the competition between the plasmodia, with the plasmodium having the strongest phenotype (having the most and quickest dominant loci) being the survivor.

\section{Non-heterothallic isolates and incompatibility}

Although non-heterothallic myxomycete isolates (generally believed to be apomicts that produce diploid myxamoebae due to blockage of meiosis during sporulation) cannot be analyzed genetically, they still have a plasmodial incompatibility system. Collins (1966) and Collins \& Clark (1968) looked at several nonheterothallic isolates of Didymium iridis and found that the different isolates would not fuse with each other, but that all of the progeny (derived from spores of the original isolate) from an isolate fused to the parental plasmodium and each other. Thus, each isolate has a particular fusion type. Betterley \& Collins (1984) examined 23 non-heterothallic isolates of $D$. iridis and found that only three of them fused. However, these three isolates were all from the same Minnesota locality, and were thus probably derived from a single strain. In 22 of the isolates, all progeny plasmodia from a particular isolate fused with each other and not with the other lines. However, one isolate (California 9) produced a few plasmodia which did not fuse with the majority of the progeny plasmodia. It was assumed that these odd plasmodia were the product of a rare crossing over event that occurred when the meiotic blockage did not function normally. They also examined one nonheterothallic isolate of each of the following species; Didymium saturnus, D. laxifilum, 
Badhamia utricalaris, and B. apiculosporum, and found the same fusion pattern as in the $D$. iridis progeny plasmodia.

Since the plasmodia incompatibility genes of the non-heterothallic isolates do not generally undergo recombination, the fusion class of a nonheterothallic plasmodium is a good indication of genetic relatedness. If two plasmodia fuse they must be phenotypically identical for the incompatibility loci, and therefore, they are likely to belong to a genetic line if they are found in the same locality. This was assumed to be the case for the Minnesota isolates in the Betterley \& Collins (1984) report.

\section{Aphanoplasmodia and Protoplasmodia}

All of the reported myxomycete plasmodial incompatibility genetic studies have been with species having a phaneroplasmodium (large pigmented reticulate mobile structure covered with a slime sheath and having rapid protoplasmic shuttle streaming). However, there are two other plasmodial types in the myxomycetes: aphanoplasmodia (large, thin, reticulate structures with protoplasmic shuttle streaming, but lacking a slime sheath and pigmentation, until it is transformed into the presporulation coralloid stage), and protoplasmodia (small un-pigmented amoeboid structures lacking protoplasmic shuttle streaming, but having a thick slime sheath) (Gray \& Alexopoulos 1968, Haskins \& Hinchee 1974, Haskins 1981). While no genetic studies have been published using aphanoplasmodia, Haskins (1990) observed plasmodial fusion in a non-heterothallic isolate of Stemonitis flavoginita, and Clark (unpublished) in a preliminary genetic analysis of a heterothallic isolate of the same species, observed fusion and non-fusion of plasmodia derived from crossed myxamoebal clones. Thus, this species is also likely to have a genetic incompatibility system similar to that found in the phaneroplasmodial species. However, in regards to protoplasmodia, Haskins (1974) has shown that identical plasmodia of the apomictic E3 strain (ATCC\#22345) of Echinostelium minutum never fuse under any conditions. On the other hand, Wollman \& Alexpoulos (1976) reported that
Licea biforis plasmodia can fuse; however, there is some dispute concerning the reality of protoplasmodia in this species (it may be a much reduced phaneroplasmodium).

\section{Conclusions}

Genetic studies on several species of myxomycetes, with reticulate phaneroplasmodia, have shown that they have a complex three tiered system which restricts fusion to between identical or nearly identical individual plasmodia. Also, the myxomycete species with a reticulate aphaneroplasmodium, apparently have a similar system since they also display fusion and nonfusion reactions. However, the myxomycete species, with small non-reticluate protoplasmodia, never undergo fusion, even when genetically identical, and thus may have a less complex system. Further studies, using sexual isolates (Clark \& Haskins 1998, Haskins et al. 2000) of Echinostelium minutum or E. coelocephalum could add valuable information to this little known plasmodial type. Myxomycete plasmodia, at least those that have a large mobile plasmodium, need to be able to form new connections between different areas of their large coenocytic cell in order to form the reticulum which is basic to this function. However, they also need to be able to maintain their existence as distinct genetic individuals so that selection and evolution can occur. This incompatibility system, in which any mixed protoplasm is lysed, may also serve to prevent the spread of plasmodial parasites between different individual plasmodia. These opposing needs and opportunities, have apparently been met by the development of a complex plasmodial incompatibility system which prevents fusion between different plasmodia unless they are essentially identical.

The three tiered incompatibility system of the phaneroplasmodia, consist of three separate, but coordinated genetic systems: membrane fusion control, rapid cytotoxic reactions, and slow cytotoxic reactions. All three genetic systems consist of a polygenic system with dominant and recessive alleles, that prevent successful fusions unless the two plasmodia are phenotyptically identical at all of the loci $(\mathrm{CC}$ and $\mathrm{Cc}$ are 
phenotypically identical, but different from cc). The mechanism that controls membrane fusion is unknown, but it may consist of protein or carbohydrate membrane components, that prevent fusion unless they are identical. The rapid cytotoxic reaction (clear zone formation) is apparently caused by pre-formed substances which exist in the plasmodia prior to fusion, and are thus activated when the cytoplasmic mixing occurs. On the other hand, the slow cytotoxic reaction requires the synthesis of RNA and protein, triggered by the mixed cytoplasm, before the reaction can occur. Since each of the three systems probably has a minimum of five loci, a species will have a minimum of 32,768 different fusion phenotypes of which 1,024 would produce immediate and obvious non-fusion reactions. Thus the probability that any two plasmodia, that are not very closely related, will undergo, or appear to undergo, a successful fusion is extremely small. This assumption has been used in several papers dealing with ecology, taxonomy, and population structure. Biosystematic studies of Didymium squamulosum (El Hage et al. 2000) and Physarum compressum (Irawan et al. 2000) were conducted using isozyme patterns and fusion classes to determine population structure and relationships. In some cases, isolates that had identical isozyme patterns could still be distinguished from each other, by non-fusion reactions. Stephenson et al. (2004) were able to determine, by fusion studies, that the plasmodia of an un-identified species (possibly Didymium nigripes), in an extensive forest area all belonged to a single genetic strain. Since very little is known about the spatial distribution and population structure of any myxomycete species, plasmodial fusion, in conjunction with DNA studies, appears to be a useful tool for exploring this problem.

\section{Acknowledgments}

This review is derived from the research of many investigators of plasmodial incompatibility. We can only hope that we have done justice to everyone's contribution to this field and have produced a useful summary for future researchers.

\section{References}

Alexopoulos CJ, Zabka GG. 1962 - Production of hybrids between physiological races of the true slime mold Didymium iridis. Nature 193, 598-599.

Betterley DA, Collins OR. 1984 - Vegetative incompatibility and myxomycete biology. Mycologia 76, 785-792.

Brandza M. 1927 - Sur la fusion ou la séparation des plasmodiums, prises comme critériums dans la definition del'espèce chez les myxomycès. Comptes Rendus Séances de l'Academe Sciences (Paris) 185, 10721074.

Border DJ, Carlile MJ. 1974 - Somatic incomepatibility following plasmodial fusion between strains of the myxo-mycete Physarum policephalum. Journal of General Microbiology 85, 211-219

Carlile MJ. 1972 - The lethal reaction following plasmodial fusion between two strains of the myxomycete Physarum policephalum. Journal of General Microbiology 71, 581590.

Carlile MJ. 1974 - Incompatibility in the myxomycete Badhamia utricularis. Transactions of the British Mycological Society 62, 401-429.

Carlile MJ. 1976 - The genetic basis of the incompatibility reaction following plasmodial fusion between different strains of the myxomycete Physarum policephalum. Journal of General Microbiology 93, 371-376.

Carlile MJ, Dee J. 1967 - Plasmodial fusion and lethal interaction between strains of a myxomycete. Nature 215, 832-834.

Cienkowski L. 1863 - Zur Entwicklungs geschichte der Myxomyceten. Prongsheim's Jabrbücher für Wissenschaftliche Botanik 3, 325-337.

Clark J. 1977 - Plasmodial incompatibility reactions in the true slime mold Physarum cinereum. Mycologia 69, 46-52.

Clark J. 1980a - Competition between plasmodial incompatibility phenol types of the myxomycete Didymium iridis. I. Paired 
plasmodia. Mycologia 72, 312-321.

Clark J. 1980b - Competition between plasmodial incompatibility phenotypes of the myxomycete Didymium iridis. II. Multipleclone crosses. Mycologia 72, 512-522.

Clark J. 1984 - Three-clone Didymium iridis crosses and plasmodial incompatibility phenotype. Mycologia 76, 810-815.

Clark J, Collins OR. 1972 - Directional cytoplasmic reactions between incomepatible plasmodia of Didymiumiridis. Genetics 73, 247-257.

Clark J, Collins OR. 1973 - Further studies on the genetics of plasmodial incompa-tibility in a Honduran isolate of Didymium iridis. Mycologia 65, 507-518.

Clark J, Collins OR. 1978 - Plasmodial incompatibility in the myxomycete Didymium nigripes. Mycologia 70, 12491253.

Clark J, Hakim R. 1980 - Nuclear sieving of Didymium iridis plasmodia. Experimental Mycology 4: 17-22.

Clark J, Haskins EF. 1998 - Heterothallic mating systems in the Echinostelium minutum complex. Mycologia 90, 382-388.

Clark J, Haskins EF. 2011 - Principles and protocols for genetical study of myxomycete reproductive systems and plasmodial coalescence. Mycosphere 2, 487-497.

Collins OR. 1966 - Plasmodial compatibility in heterothallic and homothallic isolates of Didymium iridis. Mycologia 58, 362-372.

Collins OR. 1972 - Plasmodial fusion in Physarum polycephalum: genetic analysis of a Turtox strain. Mycologia 64, 11301137.

Collins OR, Clark J. 1966 - On the genetic basis of plasmodial compatibility in Didymium iridis. American Journal of Botany 53(abstracts): 625.

Collins OR, Clark J. 1968 - Genetics of plasmodial compatibility and heterokaryosis in Didymium iridis. Mycologia 60, 90-103.

Collins OR, Haskins EF. 1970. Evidence for polygenic control of plasmodial fusion in
Physarum polycephalum. Nature 226, 279-280.

Collins OR, Haskins EF. 1972. Genetics of somatic fusion in Physarum policephalum: the PpII strain. Genetics 71, 6371.

Collins OR, Ling H. 1972. Genetics of somatic cell fusion in two isolates of Didymium iridis. American Journal of Botany 59, 337-340.

De Bary A. 1887. Comparative Morphology and Biology of the Fungi, Mycetozoa and Bacteria. Clarendon Press, London.

Dee J, Anderson RW. 1984. The effect of ploidy on the stability of plasmodial heterokaryons in Physarum policephalum. Journal of General Microbiology 131,1167-1179.

El Hage N, Little C, Clark J, Stephenson SL. 2000. Biosystematics of the Didymium squamulosum complex. Mycologia 92, 54-64.

Gray WD. 1945. The existence of physiological strains of Physarum polycephalum. American Journal of Botany 32, 157-160.

Gray WD, Alexopolos CJ. 1968 - Biology of the myxomycetes. Ronald Press, New York.

Haskins EF. 1981 - Some observations on sporulation in the myxomycete Stemonitis flavogenita. Journal of the Elisha Mitchell Science Society 97, 139-144.

Haskins EF. 1990 - Plasmodial coalescence in Stemonitis flavogenetia (Myxomycetes, Stemonitales). Mycologia 52, 643-647.

Haskins EF, Hinchee AA. 1974 - Light- and ultra-microscopical observations on the surface structure of the protoplasmodium, aphanoplasmodium, and phaneroplasmodium (Myxomycetes). Canadian Journal of Botany 52, 1835-1839.

Haskins EF, McGuinness MD, Clark J. 2000 Heterothallic mating systems in the Echinosteliales II. Echinostelium coelocephalum. Mycologia 92, 1080-1084.

Irawan B, Clark J, Stephenson SL. 2000 Biosystematics of the Physarum compressum morphospecies. Myco-logia 92, 884-893. 
Jeffery WR, Rusch HP. 1974 - Induction of somatic fusion and heterokaryosis in two incompatible strains of Physarum polycephalum. Developmental Biology 39, 331-335.

Kerr N. 1963 - The fusion of marked plasmodia of the true slime mold Didymium nigripes. Journal of Cell Biology 19(abstracts) 39.

Kerr N. 1965 - Disappearance of a genetic marker from a cytoplasmic hybrid plasmodium of a true slime mold. Journal of Protozoology 12, 276-278.

Kerr N, Waxlax JN. 1968 - The fusion of plasmodia of a true slime mould grown on various nutrients and the mixing of fused plasmodia. Transactions of the American Microscopical Society 87, 197-200.

Lane B, Carlile MJ. 1979 - Post-fusion somatic incompatibility in plasmodia of Physarum polycephalum. Journal of Cell Science 35, 339-354.

Ling H. 1972 - Genetics of somatic fusion in a myxomycete: $F_{2}$ studies. Protoplasma 73, 407-418.

Ling H, Clark J. 1981 - Somatic cell incompatibility in Didymium iridis: locus identification and function. American Journal of Botany 68, 1191-1199.

Ling H, Collins OR. 1970a - Control of plasmodial fusion in a Panamanian isolate of Didymium iridis. American Journal of Botany 57, 292-298.

Ling H, Collins OR. 1970b - Linkage studies in the true slime mold Didymium iridis. American Journal of Botany 57: 299-303.

Ling H, Ling M. 1974 - Genetic control of cell fusion in a myxomycete. Heredity $32,95-$ 104.

Ling H, Upadhyaya KC. 1974 - Cytoplasmic incompatibility studies in the myxomycete Didymium iridis: recovery and nuclear survival in heterokaryons. American Journal of 61,598-603.

Massee G. 1892 - A Monograph of the Myxogastres. Methuen, London.

McCormick JJ. 1974 - Physical separation of nuclei from two independent plasmodia of
Physarum policephalum. Journal of Cell biology 62, 227-231.

Miller DM, Anderson JD, Peterjohn GW. 1964 Protoplasmic distribution and plasmodial fusion in a myxomycete Physarum polycephalum. Transactions of the Illinois State Academy of Science 57, 94-97.

Mukherjee KL. 1965 - Plasmodial fusion reactions between three races of Didymium iridis and their hybrids. Journal of the Indian Botanical Society 44, 224230.

Poulter RTM, Dee J. 1968 - Segregation of factors controlling fusion between plasmodia of the true slime mould Physarum polycephalum. Genetic Research 12, 71-79.

Ross I, Shipley G. 1973 - Sexual and somatic fusion in the heterothallic slime mould Didymium iridis 2. Effects of actinomycin D, cyclohexamide and lysosome stabilizers. Microbios 7,165-171.

Schrauwen JAM. 1979 - Post-fusion incompatibility in Physarum policephalum. Archives of Microbiology 112, 17.

Schrauwen JAM. 1981 - Post-fusion incompatibility in Physarum policephalum. The involvement of DNA. Archives of Microbiology 129, 257-260.

Schrauwen JAM. 1985a - Post-fusion incompatibility in Physarum policphalum: changes in protein pattern of a heterokaryon. Canadian Journal of Microbiology 31, 778-781

Schrauwen JAM. 1985b - Post-fusion incompatibility in Physarum policephalum: involvement of membranes. Canadian Journal of Microbiology 31, 782-785.

Seifriz W. 1944 - Exotoxins from slime molds. Science 100, 74-75.

Skupienski FX. 1934 - Sur l'existence de races physiologiques chez les Myxomycètes. Annales de la Protistologie 4, 121-132.

Skupienski FX. 1939. Races physiologiques chez le myxomycètes Didymium squamulosum 
Fries. Comptes Rendus de la Sociéte de Biologie 131, 355-357.

Stephenson SL, Clark J, Landolt JC. 2004 Myxomycetes occurring as single genetic strains in forest soils. Systematics and Geography of Plants 74, 287-295.

Torrend C. 1907 - Les Myxomycètes 'Etude des espèce connues jusqu'ici. Brotéria 6, 5-64.

Upadhyaya KC, Ling H. 1972 - Fine structure of clear zones produced by partial intermixing of protoplasm of two phenotypically different plasmodia in a myxomycete. American Journal of Botany 59(abstracts) 6.

Upadhyaya KC, Ling H. 1976 - Ultrastructure of clear zones exhibiting cytoplasmic incompatibility following somatic cell fusion in the myxomycete Didymium iridis. Indian Journal of Experimental Biology 14, 652-658.

Wheals AE. 1970 - A homothallic strain of the myxomycete Physarum policephalum. Genetics 55, 623-631.

Wollman C, Alexopoulos CJ. 1976 - The plasmodium of Licea biforis in agar culture. Mycologia 59, 423-430. 